\title{
Heat stress affects vegetative and reproductive performance and trait correlations in tomato (Solanum lycopersicum)
}

\author{
Jiemeng Xu • Mieke Wolters-Arts • Celestina Mariani · Heidrun Huber • \\ Ivo Rieu (1)
}

Received: 13 December 2016/ Accepted: 24 June 2017 / Published online: 3 July 2017

(C) The Author(s) 2017. This article is an open access publication

\begin{abstract}
High ambient temperature has adverse effects on plant vegetative and reproductive development and reduces crop yield. To better understand the importance of male and female fertility for tomato fruit set ability under high temperature conditions and to test whether heat tolerance levels among and between reproductive and vegetative traits of genotypes correlate with each other, 13 tomato cultivars were subjected to long-term moderate heat (LTMH) or short-term heat shock (STHS), depending on the trait that was evaluated. LTMH caused significant decrease in performance of nearly all reproductive traits, i.e.
\end{abstract}

This article is part of the Topical Collection on Plant Breeding: the Art of Bringing Science to Life. Highlights of the 20th EUCARPIA General Congress, Zurich, Switzerland, 29 August-1 September 2016

Edited by Roland Kölliker, Richard G. F. Visser, Achim Walter \& Beat Boller

Electronic supplementary material The online version of this article (doi:10.1007/s10681-017-1949-6) contains supplementary material, which is available to authorized users.

J. Xu · M. Wolters-Arts · C. Mariani · I. Rieu ( $₫)$

Department of Molecular Plant Physiology, Institute for Water and Wetland Research, Radboud University, 6500 GL Nijmegen, The Netherlands

e-mail: i.rieu@science.ru.nl

\section{H. Huber}

Department of Experimental Plant Ecology, Institute for Water and Wetland Research, Radboud University, 6500 GL Nijmegen, The Netherlands pollen viability, pollen number, female fertility, seeded-fruit set and flower number per inflorescence, but not in inflorescence number. Considerable variation was found among cultivars, both under control and LTMH conditions. The cultivars Nagcarlang, Saladette and Malintka 101 produced a higher percentage of viable pollen under LTMH. For fruit set under LTMH condition, only cultivars that had been previously reported as being heat-tolerant produced fruits with seeds. STHS negatively affected vegetative traits concerning seedling survival and membrane stability. Correlation analysis revealed relationships between various traits within the control and heat treatments, but not between the two. Under heat stress fruit set was positively correlated with pollen viability, as well as with flower number per inflorescence. However, no significant correlations were found between vegetative and reproductive traits. Our data highlight the prominent role of pollen viability for tomato fertility under LTMH growth conditions. The observed variation in thermotolerance among different cultivars offers the possibility to decipher underlying physiological and genetic mechanisms.

Keywords Fruit set - Heat stress - Pollen viability . Reproduction $\cdot$ Thermotolerance $\cdot$ Tomato
Abbreviations
FF Female fertility
FPI Flower number per inflorescence 


$\begin{array}{ll}\text { FS } & \text { Fruit set } \\ \text { IL } & \text { Ion leakage } \\ \text { IN } & \text { Inflorescence number } \\ \text { LTMH } & \text { Long-term moderate heat } \\ \text { PN } & \text { Pollen number } \\ \text { PV } & \text { Pollen viability } \\ \text { SR } & \text { Seedling survival rate } \\ \text { STHS } & \text { Short-term heat stress }\end{array}$

\section{Introduction}

When plants experience ambient temperatures higher than optimal in the form of either long-term moderate heat (LTMH) or short-term heat shock (STHS), it may lead to disruption of cellular and organismal homeostasis, also known as heat stress. Basic physiological processes, such as photosynthesis, assimilate partitioning, growth and development are adversely affected (Bokszczanin et al. 2013). One of the major effects of high temperatures is the reduction of reproductive success, which commonly translates into yield loss in agricultural settings (Asseng et al. 2011). Despite the wide occurrence of this phenomenon among plant species, the underlying mechanisms are not well understood (Zinn et al. 2010; Müller and Rieu 2016; Rieu et al. 2017).

Tomato (Solanum lycopersicum) is an important horticultural crop that also functions as a research model for the plant family of Solanaceae. The optimal daily mean temperature for tomato fruit set under standard field conditions is between 21 and $24{ }^{\circ} \mathrm{C}$ (Geisenberg and Stewart 1986), but the cultivation of this crop in subtropical regions inevitably results in plants being exposed to higher day and night temperatures for successive days or even weeks during the reproductive growth phase, which can greatly hamper fruit set (Peet et al. 1997, 1998; Sato et al. 2000). Depending on the maximum day and night time temperatures, and the frequency and duration of exposure, heat has a suite of effects on reproductive development and physiology in tomato. When experiencing pre-anthesis LTMH, flowers had reduced pollen production, pollen viability, pollen shedding, ovule viability and stigma receptivity, and increased physical distance between stigma and anther cone (Kinet and Peet 1997). While all these abnormalities can occur, it is not fully clear which aspects are the most limiting for tomato fruit set under heat stress and whether tolerance levels for the various processes are related to each other. Screening of sets of tomato cultivars and wild relatives for reproduction under heat stress (LTMH or STHS) has revealed considerable, heritable natural variation in heat tolerance and several studies suggested that viability of male and female gametes, as well as the level of style protrusion are major determinants for reproductive success under these conditions, dependent on the cultivars studied (Rick and Dempsey 1969; Rudich et al. 1977; Levy et al. 1978; Dane et al. 1991; Saeed et al. 2007; Bhattarai et al. 2016).

Here, we evaluated the reproductive traits in a diverse set of tomato cultivars under control and LTMH condition, and the vegetative traits under control and STHS condition, in order to (1) assess the importance of male and female fertility in determining tomato fruit set under LTMH conditions (2) test whether heat tolerance levels among and between reproductive and vegetative traits correlate with each other, and (3) identify genotypes with distinctively contrasting heat stress performance for further studying the genetic and physiological differences underlying variation in reproductive heat tolerance.

\section{Materials and methods}

\section{Plant material}

Thirteen inbred cultivars of tomato (Solanum lycopersicum) were obtained from various sources (Table S1). Five of them were explicitly reported to be heat tolerant regarding fruit set, while for eight cultivars, two commercial lines and six more regularly used lines, no information with respect to heat tolerance was publically available (Table S1).

Determining reproductive performance under LTMH conditions

Tomato seeds were sown in standard potting compost (Lentse Potgrond number 4, Horticoop B.V., Katwijk, The Netherlands) and covered with vermiculite. At 10 days, seedlings were transferred into separate small pots and at 20 days seedlings were transplanted into 12-L pots filled with the same potting compost supplemented with slow-release fertilizer $\left(4 \mathrm{~g} \mathrm{~L}^{-1}\right.$ Osmocote Exact Standard 3-4 M, Everris International 
B.V., Geldermalsen, The Netherlands). Plants were grown under standard greenhouse conditions with 16-h light period (supplemented with artificial light from $600 \mathrm{~W}$ sodium lamps if natural light intensity fell below $250 \mu \mathrm{mol} \mathrm{m} \mathrm{m}^{-2} \mathrm{~s}^{-1}$ ) and temperature of about $25^{\circ} \mathrm{C}$ during the day (minimum set to $20^{\circ} \mathrm{C}$ ) and $19{ }^{\circ} \mathrm{C}$ at night (minimum set to $17^{\circ} \mathrm{C}$ ). When the first inflorescences were detectable by eye, all inflorescences were removed and plants were transferred to climate chambers with LTMH conditions (14 h light period with intensity of $\sim 250 \mu \mathrm{mol} \mathrm{m} \mathrm{m}^{-2} \mathrm{~s}^{-1}$ at plant height from Phillips $600 \mathrm{~W}$ SON-T lamps; $32{ }^{\circ} \mathrm{C}, 70 \%$ $\mathrm{RH}$, VPD $1.428 \mathrm{kPa} / 26^{\circ} \mathrm{C}, 80 \% \mathrm{RH}$, VPD $0.673 \mathrm{kPa}$ during day/night, with temperature fluctuation $<1{ }^{\circ} \mathrm{C}$ ) or control conditions (equal light and $\mathrm{RH} ; 25^{\circ} \mathrm{C}$, VPD $0.951 \mathrm{kPa} / 19{ }^{\circ} \mathrm{C}$, VPD $0.440 \mathrm{kPa}$ during day/night). Two weeks later, the percentage of pollen that were viable (PV) and pollen number (PN) were evaluated on newly formed flowers as described by Rodriguez-Riano and Dafni (2000). In brief, anther cones were cut into 4 pieces and pollen were released into staining buffer consisting of peroxidase indicator (Sigma 3901-10VL), $200 \mu \mathrm{L} \mathrm{3 \%} \mathrm{H}_{2} \mathrm{O}_{2}$, and $50 \mathrm{~mL} 10$ times diluted Trizmal buffer (903C; Sigma-Aldrich, St Louis, MO, USA) by vortexing. The resulting pollen suspension was incubated for $20 \mathrm{~min}$ at $37{ }^{\circ} \mathrm{C}$ and loaded onto a haemocytometer. Dark stained pollen was considered as viable. Per flower approximately 100 pollen were assessed for PV analysis. The number of pollen in 25 squares $(0.1 \mu \mathrm{L})$ of the haemocytometer was counted and converted to $\mathrm{PN}$ based on total resuspension volume. In addition, inflorescence number (IN; i.e. from the whole plant) and flower number per inflorescence (FPI; 3 inflorescences per plant) were recorded. To determine the percentage of seeded-fruit set (FS), flowers (5-10 per plant) were tagged and self-pollinated by mechanical vibration and kept in $\mathrm{CMH}$ or control conditions for one more week before being transferred back to the standard greenhouse conditions. In addition, immediately after relocation to the greenhouse, 5 flowers per plant were pollinated with pollen from control treatment to determine female fertility (FF). Per cultivar and treatment, 2-5 plants were analysed.

Evaluating vegetative thermotolerance under STHS conditions

The heat tolerance of tomato cultivars at vegetative stage was determined by seedling survival rate and ion leakage assay. Seeds of the same cultivars used in the reproductive phenotyping experiment were sown in trays filled with potting compost as described above. In order to obtain seedlings of similar developmental stage, sowing date was adjusted up to 2 days per cultivar. After sowing, the trays were kept in a growth cabinet $\left(25 / 19{ }^{\circ} \mathrm{C}\right.$, day/night; $12 \mathrm{~h}$ light with intensity of $\sim 250 \mu \mathrm{mol} \mathrm{m} \mathrm{m}^{-2} \mathrm{~s}^{-1}$ at plant level supplied by Philips Green Power LED DR/B/FR 120 lamps, $12 \mathrm{~h}$ dark; $60 \% \mathrm{RH}$ ) for 12 days. Uniformly developed seedlings with the first and second true leaf visible were subjected to a STHS treatment $\left(50{ }^{\circ} \mathrm{C}\right)$ treatment for $6 \mathrm{~h}$, in the dark. After recovery at control conditions for 1 week, seedlings with shrunken and dying stem beneath the apical meristem were considered as dead, and thereon seedling survival rates (SR) was calculated. The whole experiment was repeated three times (19-62 seedlings per cultivar per time).

To evaluate the membrane stability, leaf discs were used for leakage assay described as Camejo et al. (2005). The conditions for seed germination, seedling growth were maintained the same as reproductive phenotyping. Before emergence of the 1st truss, 9 leaf discs were collected from the 3rd to 5th fully developed leaf (counted from the top to the bottom). Leaf discs were washed with deionized water three times for 5 min per time. Cleaned leaf samples were transferred to $50 \mathrm{~mL}$ tubes filled with $15 \mathrm{~mL}$ deionized water and incubated at STHS $\left(42{ }^{\circ} \mathrm{C}\right.$ ) condition for $3 \mathrm{~h}$. For each tube conductivity was measured twice, i.e. immediately after cooling down to room temperature ("E1") and $1 \mathrm{~h}$ later ("E2"), and again after disrupting the leaf cells by the incubation at $100{ }^{\circ} \mathrm{C}$ for $1 \mathrm{~h}$ ("E3"). Ion leakage (IL) was calculated as $(\mathrm{E} 1+\mathrm{E} 2 / 2) / \mathrm{E} 3$. The experiment was repeated three times ( 3 plants per cultivar per replicate).

\section{Statistical analysis}

PV, FS, SR and IL data were logit transformed and PN and FF data were log transformed before analysis to improve the normality and reduce the heteroscedasticity of the data. The overall effects of treatment and cultivar and their interaction were analysed by means of two-way ANOVA with heat treatment and cultivar as fixed factors. Cultivars were treated as fixed factors because we were interested in the response of the specific cultivars used in this experiment. In order to infer which specific cultivars are sensitive or 
insensitive to heat conditions, a Student's $t$ test was performed for each cultivar separately. In order to be get more information on within treatment genetic variation among cultivars (i.e. to see which specific cultivars differ from each other), a one-way ANOVA followed by post hoc Tukey comparisons was performed for plants grown under control or heat separately. For the analyses of FS was a non-parametric Kruskal-Wallis Test was used because even after transformation the distributions of the values for this trait were not conform to the assumptions of parametric tests. Prior to the analyses, the plant mean value of treats measured on several flowers for each plant (PV, PN, IN, FPI, FF, FS) or time (IL, SR) were calculated. To illustrate whether heat (LTMH for reproductive traits, STHS for vegetative traits) influenced the proportion of variance explained by the different components (individual plants, cultivar), data were separated in heat and control groups. Sum of squares from each factor were calculated with a nested ANOVA design in order to be able to differentiate between the within plant variance (i.e. variation among flowers produced by a single plant, i.e. for PV, PN, IN, FPI, FF, FS), among plant variance (i.e. variation among plant replicates from a single cultivar) and among cultivar variance. Sum of squares of each component were divided by total sum of squares to calculate explained variance. While within and among plant variance may be mainly due to breaking up of genetic correlations and disruption of developmental processes during stress conditions, increased among cultivar variance may provide breeders with the opportunity to select genotypes which are better adapted to high temperature conditions. In this analysis, e.g. a relatively higher proportion of the variance attributed to cultivar in heat as compared to control treatments would indicate that the different cultivars displayed a greater variation in heat as compared to control treatments, i.e. that they differentiate with respect to heat susceptibility. To explore relationships among traits, Pearson correlation coefficients were calculated on the mean values for each cultivar. If not explicitly mentioned otherwise, all statistical analyses were done with SPSS v.20 (IBM, NY, USA).

\section{Results}

To describe natural variation for tolerance to heat conditions and detect correlations between traits, we analysed reproductive traits of 13 tomato cultivars under a control temperature profile and long-term mild heat (LTMH) and vegetative traits under control and short-term heat shock (STHS) (Table S1).

\section{Male fertility}

To assess male reproductive performance, pollen viability (PV) and pollen number at flower anthesis were analysed under control and LTMH conditions. Overall, PV was significantly decreased by LTMH treatment, and cultivar differences were found both, in control and LTMH (Table 1; Table S2). There was a significant interaction between cultivar and treatment (Table 1), indicating that not all cultivars responded similarly to heat. Under control conditions, PV ranged from 47 to $83 \%$ and under LTMH from 3.9 to $31 \%$, with the cultivars Nagcarlang, Malintka 101 and Saladette showing relatively high PV under LTMH condition compared to the other cultivars.

Less variation among cultivars was observed for PN (Table 1; also see Fig. 1). All 13 cultivars had similar PN under control condition. As for LTMH, most of the cultivars produced fewer pollen, except for cultivars NCHS-1 and Hotset, explaining the significant interaction between cultivar and treatment for this trait (Table 1).

\section{Female fertility}

Female fertility was determined by evaluating seed set upon manual pollination of freshly opened flowers from LTMH conditions with pollen that developed under control conditions, with pollination and fruit set taking place under control conditions. Genotypic variation was observed in both temperature conditions, but particularly in LTMH (Table 1; also see Fig. 1). An overall significant, negative effect of LTMH on FF was found; at cultivar level the reduction in FF in response to LTMH was significant for Malintka 101, Hotset, Micro-Tom and Pull (Table 1). Hotset, Nagcarlang and F1 Ninja were the three cultivars that maintained highest FF under LTMH conditions (Table 1).

\section{Fruit set ability}

Fruit set (FS), as indicated by the percentage of seeded fruits produced upon mechanical self-pollination, was 


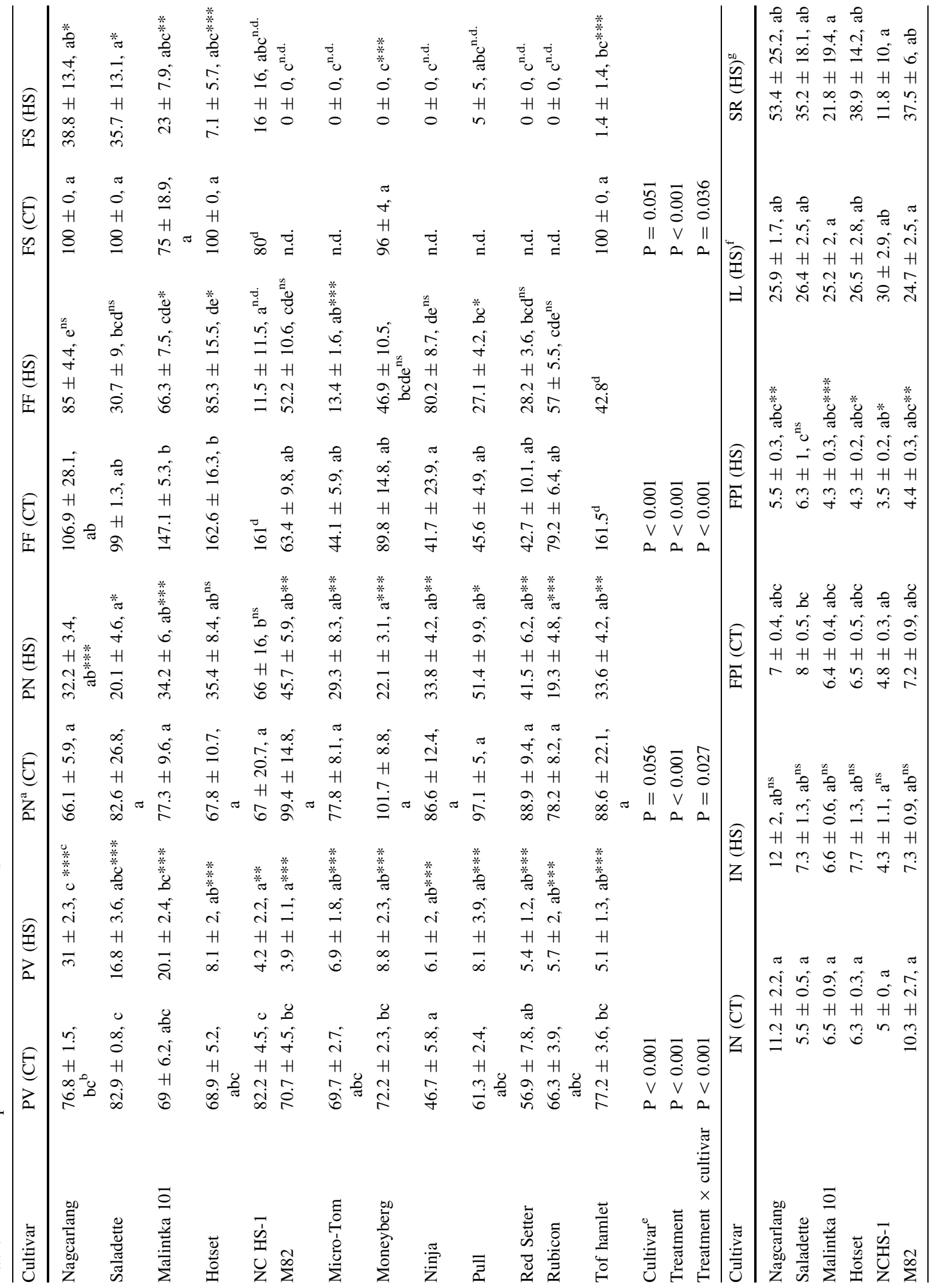




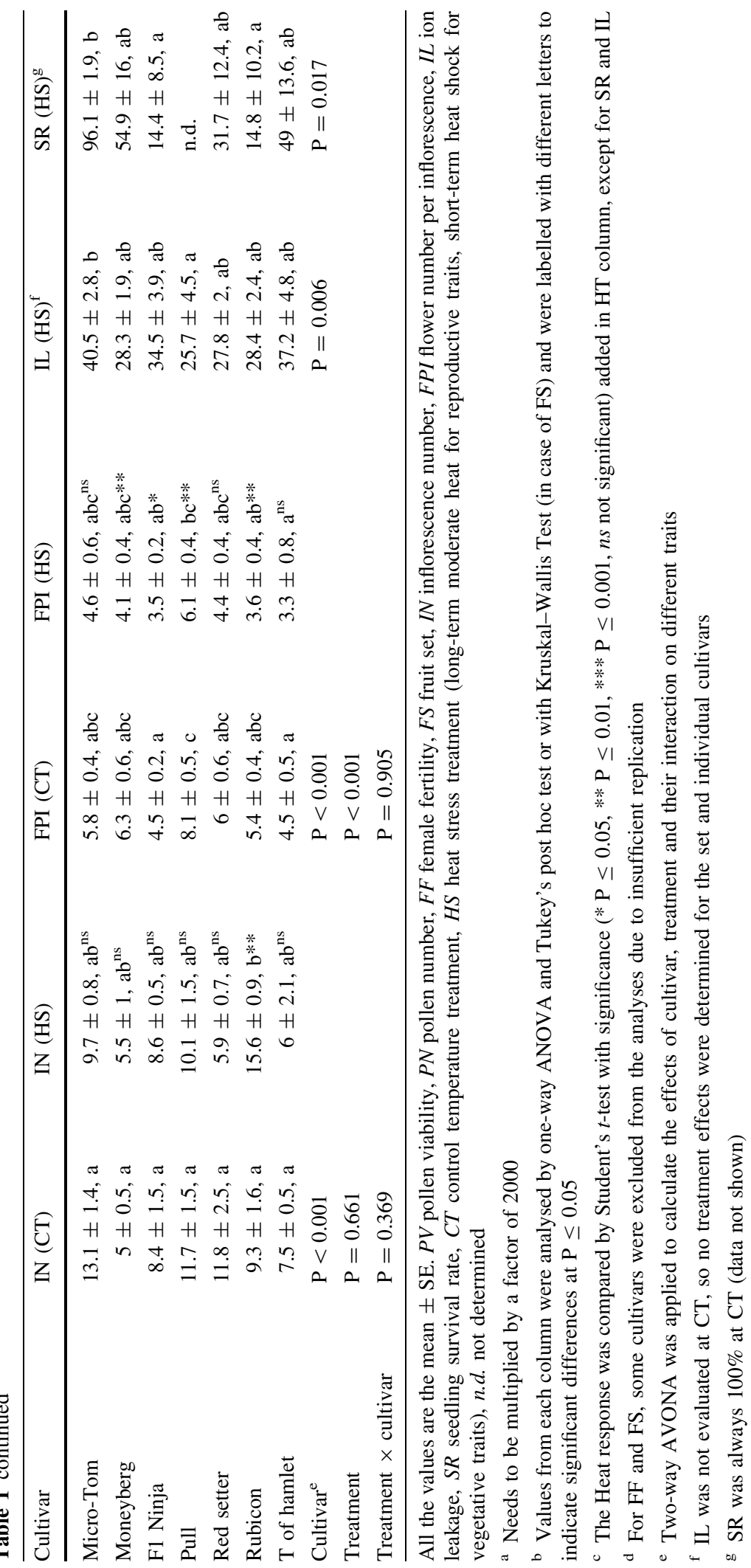




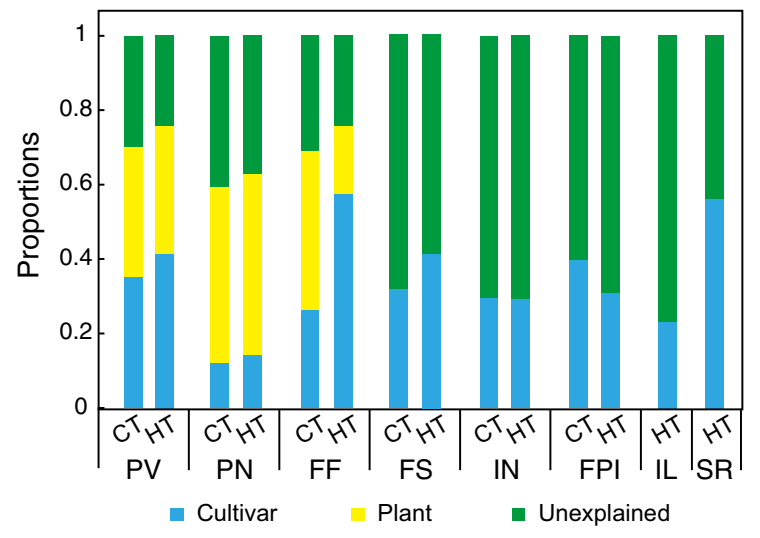

Fig. 1 Proportions of total variance explained by different factors. Plants were phenotyped under control (CT) and heat stress (HS) conditions (long-term moderate heat, LTMH, for reproductive traits, short-term heat shock, STHS, for vegetative traits). Plant-level explained variance could be estimated for PV, $\mathrm{PN}$ and FF, only. Trait abbreviations, $P V$ pollen viability, $P N$ pollen number, $F F$ female fertility; $F S$ fruit set, $I N$ inflorescence number, FPI flower number per inflorescence, $I L$ ion leakage, $S R$ seedling survival rate

assessed in a subset of cultivars under LTMH conditions. No cultivar differences in FS were detected under control conditions. The LTMH treatment drastically reduced FS, with only few cultivars producing seeded fruits (Table 1). Thus, there was a very significant, negative effect of temperature treatment, in addition to a marginally significant cultivar effect, and a significant interaction between cultivar and temperature treatment (Table 1).

\section{Flowering behaviour}

Two flowering-related developmental traits, inflorescence number (IN) and the number of flowers per inflorescence (FPI) were recorded under both control and LTMH conditions. Overall, IN was not affected by the LTMH treatment (Table 1). A significant cultivar difference was only found in LTMH conditions, with the cultivar Rubicon having a higher IN than NCHS-1.

On average, LTMH decreased FPI by $28 \%$ (Table 1). FPI differed significantly among cultivars under both growing conditions, but no difference in response was found among cultivars (Table 1).

\section{Vegetative performance}

In addition to thermotolerance evaluation at the reproductive phase, heat-tolerance of seedlings and membrane stability of leaf cells were determined. As seedling growth was not visibly affected by LTMH, seedling heat tolerance was assessed as survival rate (SR) after exposure to a STHS of $50{ }^{\circ} \mathrm{C}$ for $6 \mathrm{~h}$. While SR was $100 \%$ under control for all cultivars, the STHS treatment significantly reduced seedling survival (Table 1). Differences among cultivars were evident, with SR ranging from 12 to $96 \%$, with Micro-Tom performing best.

Ion leakage (IL) of leaf tissue was used as a proxy for membrane integrity, where higher leakage represents lower membrane integrity. Again, as LTMH did not affect IL substantially, we used a single STHS incubation, at $42{ }^{\circ} \mathrm{C}$ for $3 \mathrm{~h}$, and found significant differences in IL among cultivars (Table 1). The cultivars, M82, Malintka 101 and Pull kept relatively high membrane integrity upon the STHS treatment, while Micro-Tom had the lowest (Table 1).

\section{Trait variation in a set of tomato cultivars}

To determine the contribution of genetic and environmental factors to the phenotype, the proportions of phenotypic variance that could be explained by cultivar and plant effects were calculated for control and heat conditions separately (Fig. 1). Pollen number (PN) was relatively stable across cultivars, leading to a low genetic component of about $10 \%$ of the total variance but was characterized by the highest among plant within cultivar variance (approximately 50\%). For the other traits the contribution of the cultivar to the total variance ranged from 30 to $60 \%$. The lowest among plant variance $(10 \%)$ was found for female fertility (FF) of plants subjected to LTMH. Overall, heat hardly affected the allocation of variance components except for FF. In plants subjected to LTMH, the among plant variance decreased from 40 to $10 \%$ while the among cultivar variance increased from 25 to $55 \%$, indicating that the cultivars did differ with respect to heat sensitivity while the overall development appeared to be more canalized under higher temperature.

\section{Correlations between traits}

To determine relationships among the aforementioned traits, Pearson correlation coefficients were calculated among those analysed traits from control and heat conditions (LTMH or STHS for reproductive traits and 


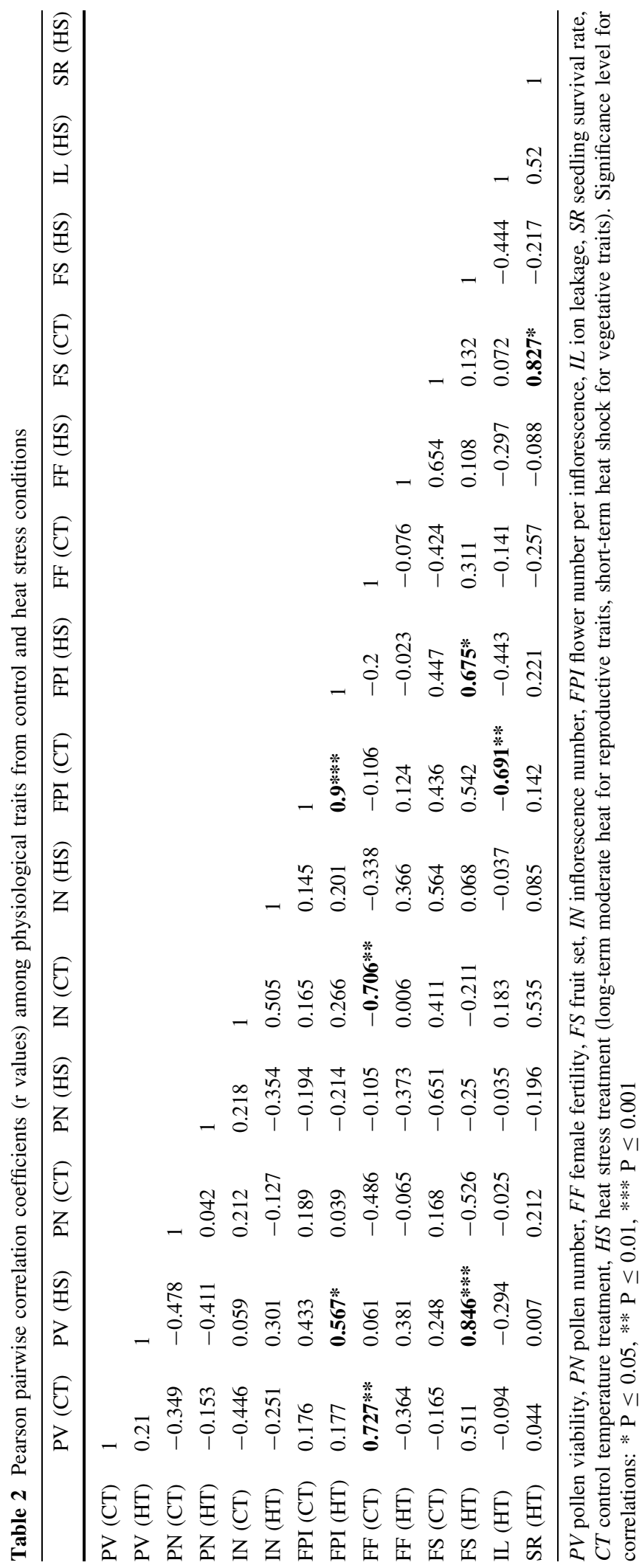


vegetative traits, respectively) (Table 2). This analysis revealed correlation clusters mainly within temperature treatment. Under control conditions, PV and FF were positively correlated with each other, while FF was negatively correlated with IN. In the heat treatment, positive correlations were found between each pair of the 3 traits: FS, PV and FPI. Furthermore, FPI from control condition was positively correlated with FPI from LTMH and negatively with IL from STHS. FF under HT did not associate with any other traits. There was no significant correlation between trait means of cultivars subjected to control conditions and their heat treatment response.

\section{Discussion}

Heat stress affects reproductive traits, dependent on genotype

At the reproductive stage, long-term mild heat (LTMH) had significantly harmful effects on pollen viability (PV), pollen number (PN) and female fertility (FF), which is in line with other studies (Levy et al. 1978; Peet et al. 1998; Sato et al. 2000; Pressman et al. 2002; Firon et al. 2006; Sato et al. 2006; Paupière et al. 2017). The responses of different traits to the heat varied, with an average reduction of 86,56 and $39 \%$ for PV, PN and FF, respectively. For each of the 13 cultivars, reduction in PV was larger than that in FF, which strengthens the notion that development of the male gametophyte is more sensitive to heat than that of the female one (Peet et al. 1998). In addition to the effect of heat, analysis showed significant genotypic effects on reproductive traits. In our experiment, the three cultivars previously reported as being heattolerant, i.e. Nagcarlang, Malintka 101 and Saladette (Rudich et al. 1977; Abdul-Baki 1991; Dane et al. 1991; Opeña et al. 1992; Chetelat 2015), produced pollen with a relatively high viability when flowers completely developed under LTMH. Hotset did not perform well in this respect, but contrasting results have been reported before for this cultivar (Levy et al. 1978; Dane et al. 1991). Cultivars also differed regarding the response of PN to the LTMH. Here, cultivar NCHS-1 stood out in a positive way, as PN was not affected by temperature and was the highest of all cultivars under LTMH conditions. Regarding FF, large variation among cultivars was observed under both control and LTMH temperature regimes. Several cultivars were hardly affected by LTMH in this respect, including Nagcarlang and F1 Ninja, which together with Hotset maintained the highest FF under LTMH condition. Taken together, LTMH as well as cultivar influenced reproductive trait performances, often in interaction with each other.

Pollen viability limits fruit set under LTMH conditions

Living organisms function as physiologically integrated networks, meaning that not all traits can behave independently. There can be trade-offs, for example due to limited resources, but also dependencies, such as the requirement for viable pollen to produce fruits and seeds. In the present study, seeded-fruit set (FS) was the most strongly affected trait by the LTMH treatment, which fits with the idea that reproductive success depends on multiple heat-sensitive sub-traits, leading to a synergistic, stronger effect on final fertility. Because of the compound nature of FS and potential interactions between sub-traits, it is difficult to determine the relative contribution of each in the limited set of genotypes used in this study. For example, although the cultivar Nagcarlang produced pollen with high viability under LTMH conditions, FS was likely to be limited by the fact that its style protruded out of the anther cone, thereby impeding pollination (unpublished data; Dane et al. 1991). This complexity is also reflected by the low heritability of FS under high temperature, while the heritability of more simple sub-traits, like style exertion, is relatively high (Levy et al. 1978). Still, within our set of cultivars we found a positive correlation between FS and PV, but not FF, under LTMH conditions. As in other studies using cultivar sets, the breeding history of the cultivars used here is unknown, meaning that identified trait correlations may point at physiological dependencies or similarities, but could also reflect linkage due to genetic relationships among cultivars. However, the fact that correlation between PV and FS under LTMH conditions has now been reported multiple times with different sets of tomato cultivars (Levy et al. 1978; Dane et al. 1991; Akhtar et al. 2012) suggests that male fertility is indeed a key determinant for reproductive heat tolerance in this species. This is further corroborated by two studies in which more negative effects on reproductive success were found if 
heat was applied to the male than to the female parental plants of a cross (Levy et al. 1978; Peet et al. 1998). Similar conclusions were drawn for other plant species, such as bean, cowpea, groundnut, brachypodium, barley and rice (Ahmed et al. 1992; Prasad et al. 1999; Suzuki et al. 2000; Sakata et al. 2000; Harsant et al. 2013). The contrasting cultivars identified here may be used to dissect the physiological basis for pollen heat sensitivity. Various hypotheses have been posed (Müller and Rieu 2016; Rieu et al. 2017), most recently that anther cells suffer from a loss of male identity under long term mild heat (Müller et al. 2016). Regarding FF, considerable genotypic variation was present in the cultivar set, especially under LTMH, so the absence of a significant correlation with FS suggests that female heat tolerance is not limiting reproduction under the applied LTMH temperature regime. This corresponds to the relatively low effects of heat on the female side found in reciprocal crosses (Peet et al. 1998), but has not yet been reported in a correlative study with multiple cultivars as performed here. Under control temperature, no positive correlation between either PV or FF and FS was found, which suggests that male and female fertility are not the main limiting factors for reproduction under more optimal temperature growth conditions.

Correlations between vegetative and reproductive traits

It was reported that total flower production in tomato was reduced upon experience of severely high temperature conditions (El Ahmadi and Stevens 1979), but under moderate heat conditions, flower number seems not to be affected (Peet et al. 1997, 1998; Sato et al. 2004, 2006). In our cultivar set, LTMH did not affect the number of inflorescences (IN), but reduced the number of flowers per inflorescence (FPI), the latter of which was also found by Adams et al. (2001). This may suggest that the total flower number is compensated by longer flowering or a higher inflorescence production at later stages, which were not assayed here. Interestingly, FPI was positively correlated with FS and PV under LTMH. Similar correlations have been described by AbdulBaki (1991), who reported that genotypes that were more heat-tolerant regarding fruit set had more flowers in control and high temperature conditions. Kugblenu et al. (2013) found that under moderate heat cultivars with higher FPI showed lower incidence of flower abortion, which likely corresponds to fewer unfertilised flowers, and Akhtar et al. (2012) reported a positive correlation between FPI and PV under moderate heat conditions. Currently, it is not known what the physiological reason is for the apparent correlation between FPI and reproductive heat tolerance.

The various cultivars were also evaluated for vegetative heat tolerance traits under STHS conditions, such as thermo-stability of leaf-cell membranes. We found that the level of ion leakage through the membrane negatively linked to the FPI-FS-PV trait cluster under LTMH conditions, and significantly so via FPI under control conditions. In line with this, Camejo et al. (2005) showed that Nagcarlang had more stable membranes than a heat-sensitive cultivar. Membrane thermo-stability has often been linked to photosynthetic and respiratory performance under heat (Wahid et al. 2007), but these latter two traits do not seem to be affected much by moderate heat regimes (Sato et al. 2000; Jiang et al. 2017; Rieu et al. 2017), suggesting a different reason for the positive link between membrane stability and reproductive heat tolerance. By contrast, we did not find any relation between heat tolerance of seedlings and reproductive processes. The finding that Micro-Tom seedlings, with relatively short and thick hypocotyls, were most heat-tolerant suggests that morphological characteristics might be important at this stage.

\section{Conclusion}

Considerable natural variation for reproductive and non-reproductive traits under heat stress conditions was found. The results show that pollen viability is a major factor limiting tomato fruit set under LTMH conditions and should be a target for further studies. Furthermore, there are indications that flower number per inflorescence and membrane thermo-stability are also relevant characteristics and might be used as indicators of reproductive heat tolerance. However, the validity of potential markers would need to be confirmed for specific genetic backgrounds. Whether there is a physiological basis for these correlations or whether the traits each have a unique, but genetically linked basis remains to be investigated. Analysis of the genetic architecture behind important sub-traits may 
be pursuit using crosses between specific contrasting cultivars, such as Nagcarlang and NCHS-1 in case of pollen thermotolerance (Xu et al. 2017). The suitability of Nagcarlang for such a study is supported by the finding that it was among the best performing genotypes regarding pollen fertility and fruit set under high temperature field conditions and general combining ability of these traits (Dane et al. 1991; Bhattarai et al. 2016).

Acknowledgements The authors wish to thank Gerard van der Weerden (Experimental Garden and Genebank, Radboud University, Nijmegen, The Netherlands) and the greenhouse staff for taking excellent care of the plants. This work was supported by the China Scholarship Council (Grant Number 201207565002, to JX), the Dutch Topsector Horticulture and Starting Materials (grant number 2013-H320, to IR), the Netherlands Organisation for Scientific Research (NWOALW, grant number 867.15.011, to IR) and the European Commission (Marie Curie Initial Training Network: Solanaceae Pollen Thermotolarance/SPOT-ITN, grant number 289220, to IR).

Author contributions JX and IR conceived and designed the study, JX and MW acquired the data, JX, HH, and IR analysed and interpreted the data. JX and IR wrote the manuscript, $\mathrm{HH}$ and $\mathrm{CM}$ advised on the manuscript.

\section{Compliance with ethical standards}

Conflict of interest The authors declare that they have no conflict of interest.

Ethical standards This article does not contain any studies with human participants or animals performed by any of the authors.

Open Access This article is distributed under the terms of the Creative Commons Attribution 4.0 International License (http:// creativecommons.org/licenses/by/4.0/), which permits unrestricted use, distribution, and reproduction in any medium, provided you give appropriate credit to the original author(s) and the source, provide a link to the Creative Commons license, and indicate if changes were made.

\section{References}

Abdul-Baki AA (1991) Tolerance of tomato cultivars and selected germplasm to heat stress. J Am Soc Hortic Sci 116:1113-1116

Adams S, Cockshull KE, Cave CRJ (2001) Effect of temperature on the growth and development of tomato truits. Ann Bot 88:869-877. doi:10.1006/anbo.2001.1524

Ahmed FE, Hall AE, DeMason DA (1992) Heat injury during floral development in cowpea (Vigna unguiculata, Fabaceae). Am J Bot 79:784. doi:10.2307/2444945
Akhtar S, Ansary SH, Dutta AK et al (2012) Crucial reproductive characters as screening indices for tomato (Solanum lycopersicum) under high temperature stress. J Crop Weed 8:114-117

Asseng S, Foster I, Turner NC (2011) The impact of temperature variability on wheat yields. Glob Change Biol 17:997-1012. doi:10.1111/j.1365-2486.2010.02262.x

Bhattarai U, Sharma A, Das R, Talukdar P (2016) Genetic analysis of yield and yield-attributing traits for high temperature resistance in tomato. Int J Veg Sci 5260:1-13. doi:10.1080/19315260.2015.1118421

Bokszczanin KL, Fragkostefanakis S, Solanaceae Pollen Thermotolerance Initial Training Network (SPOT-ITN) Consortium (2013) Perspectives on deciphering mechanisms underlying plant heat stress response and thermotolerance. Front Plant Sci 4:315. doi:10.3389/fpls.2013.00315

Camejo D, Rodríguez P, Angeles Morales M et al (2005) High temperature effects on photosynthetic activity of two tomato cultivars with different heat susceptibility. J Plant Physiol 162:281-289. doi:10.1016/j.jplph.2004.07.014

Chetelat RT (2015) Revised list of miscellaneous stocks. TGRC. http://tgrc.ucdavis.edu/Misc-stocks\%20list\%202015.pdf. Accessed 6 Dec 2016

Dane F, Hunter AG, Chambliss OL (1991) Fruit set, pollen fertility, and combining ability of selected tomato genotypes under high-temperature field conditions. J Am Soc Hortic Sci 116:906-910

El Ahmadi AB, Stevens MA (1979) Reproductive responses of heat-tolerant tomatoes to high temperatures. J Am Soc Hortic Sci 104:686-691

Firon N, Shaked R, Peet MM et al (2006) Pollen grains of heat tolerant tomato cultivars retain higher carbohydrate concentration under heat stress conditions. Sci Hortic 109:212-217. doi:10.1016/j.scienta.2006.03.007

Geisenberg C, Stewart K (1986) Field crop management. In: Atherton J, Rudich J (eds) The tomato crop: a scientific basis for improvement. Springer, Netherlands, Dordrecht, pp 511-557

Harsant J, Pavlovic L, Chiu G et al (2013) High temperature stress and its effect on pollen development and morphological components of harvest index in the $\mathrm{C} 3$ model grass Brachypodium distachyon. J Exp Bot 64:2971-2983. doi:10.1093/jxb/ert142

Jiang J, Liu X, Liu C, Liu G, Li S, Wang L (2017) Integrating omics and alternative splicing reveals insights into grape response to high temperature. Plant Physiol 173:1502-1518. doi:10.1104/pp.16.01305

Kinet JM, Peet MM (1997) Tomato. In: Wien HC (ed) The physiology of vegetable crops. Cab International, Wallingford, UK, pp 207-258

Kugblenu YO, Oppong Danso E, Ofori K, Andersen MN, Abenney-Mickson S, Sabi EB, Plauborg F, Abekoe MK, Ofosu-Anim J, Ortiz R, Jørgensen ST (2013) Screening tomato genotypes for adaptation to high temperature in West Africa. Acta Agric Scand Sect B Soil Plant Sci 63:516-522. doi:10.1080/09064710.2013.813062

Levy A, Rabinowitch HD, Kedar N (1978) Morphological and physiological characters affecting flower drop and fruit set of tomatoes at high temperatures. Euphytica 27:211-218. doi:10.1007/BF00039137 
Müller F, Rieu I (2016) Acclimation to high temperature during pollen development. Plant Reprod 29:107-118. doi:10. 1007/s00497-016-0282-x

Müller F, Xu J, Kristensen L, Wolters-Arts M, de Groot PFM, Jansma SY et al (2016) High-temperature-induced defects in tomato (Solanum lycopersicum) anther and pollen development are associated with reduced expression of B-Class floral patterning genes. PLoS ONE 11:e0167614. doi:10.1371/journal.pone.0167614

Opeña RT, Chen JT, Kuo CG, Chen HM (1992) Genetic and physiological aspects of tropical adaptation in tomato. In: Adaptation of food crops to temperature and water stress: proceedings of an international symposium, pp 13-18

Paupière MJ, van Haperen P, Rieu I, Visser RGF, Tikunov YM, Bovy AG (2017) Screening for pollen tolerance to high temperatures in tomato. Euphytica 213:130. doi:10.1007/ s10681-017-1927-z

Peet MM, Willits DH, Gardner R (1997) Response of ovule development and post-pollen production processes in male-sterile tomatoes to chronic, sub-acute high temperature stress. J Exp Bot 48:101-111. doi:10.1093/jxb/48.1. 101

Peet MM, Sato S, Gardner RG (1998) Comparing heat stress effects on male-fertile and male-sterile tomatoes. Plant Cell Environ 21:225-231. doi:10.1046/j.1365-3040.1998. 00281.x

Prasad PVV, Craufurd PQ, Summerfield RJ (1999) Fruit number in relation to pollen production and viability in groundnut exposed to short episodes of heat stress. Ann Bot 84:381-386. doi:10.1006/anbo.1999.0926

Pressman E, Peet MM, Pharr DM (2002) The effect of heat stress on tomato pollen characteristics is associated with changes in carbohydrate concentration in the developing anthers. Ann Bot 90:631-636. doi:10.1093/ aob/mcf 240

Rick CM, Dempsey WH (1969) Position of the stigma in relation to fruit setting of the tomato. Bot Gaz 130:180-186

Rieu I, Twell D, Firon N (2017) Pollen development at high temperature: from acclimation to collapse. Plant Physiol 173:1967-1976. doi:10.1104/pp.16.01644
Rodriguez-Riano T, Dafni A (2000) A new procedure to assess pollen viability. Sex Plant Reprod 12:241-244. doi:10. 1007/s004970050008

Rudich J, Zamski E, Regev Y (1977) Genotypic variation for sensitivity to high temperature in the tomato: pollination and fruit set. Bot Gaz 138:448-452

Saeed A, Hayat K, Khan AA, Iqbal S (2007) Heat tolerance studies in tomato (Lycopersicon esculentum Mill.). Int $\mathrm{J}$ Agric Biol 9:649-652

Sakata T, Takahashi H, Nishiyama I, Higashitani A (2000) Effects of high temperature on the development of pollen mother cells and microspores in barley (Hordeum vulgare L.). J Plant Res 113:395-402. doi:10.1007/PL00013947

Sato S, Peet MM, Thomas JF (2000) Physiological factors limit fruit set of tomato (Lycopersicon esculentum Mill.) under chronic, mild heat stress. Plant Cell Environ 23:719-726. doi:10.1046/j.1365-3040.2000.00589.x

Sato S, Peet MM, Gardner RG (2004) Altered flower retention and developmental patterns in nine tomato cultivars under elevated temperature. Sci Hortic 101:95-101. doi:10.1016/ j.scienta.2003.10.008

Sato S, Kamiyama M, Iwata T et al (2006) Moderate increase of mean daily temperature adversely affects fruit set of $L y$ copersicon esculentum by disrupting specific physiological processes in male reproductive development. Ann Bot 97:731-738. doi:10.1093/aob/mcl037

Suzuki K, Takeda H, Tsukaguchi T, Egawa Y (2000) Ultrastructural study on degeneration of tapetum in anther of snap bean (Phaseolus vulgaris L.) under heat stress. Sex Plant Reprod 13:293-299. doi:10.1007/s004970100071

Wahid A, Gelani S, Ashraf M, Foolad M (2007) Heat tolerance in plants: an overview. Environ Exp Bot 61:199-223. doi:10.1016/j.envexpbot.2007.05.011

Xu J, Driedonks N, Rutten MJM, Vriezen WH, de Boer GJ, Rieu I (2017) Mapping quantitative trait loci for heat tolerance of reproductive traits in tomato (Solanum lycopersicum). Mol Breed 37. doi: 10.1007/s11032-017-0664-2

Zinn KE, Tunc-Ozdemir M, Harper JF (2010) Temperature stress and plant sexual reproduction: uncovering the weakest links. J Exp Bot 61:1959-1968. doi:10.1093/jxb/erq053 